\title{
A Low Power / Low Voltage Electrostatic ACtuator for RF MEMS APPLiCATIONS
}

\author{
J. Jason Yao*, SangTae Park, Robert Anderson, and Jeffrey DeNatale \\ Rockwell Science Center, 1049 Camino Dos Rios, Thousand Oaks, CA 91360 \\ *Voice: (805) 373-4471, FAX: (805) 373-4869, E-Mail: jjyao@rsc.rockwell.com
}

\begin{abstract}
We have designed and fabricated a low power / low voltage electrostatic actuator, and demonstrated its application to a large tuning-ratio tunable capacitor for RF MEMS applications. Using adhesive bonding and deep silicon reactive ion etching techniques, the entire MEMS device is made of single crystal silicon, and is suspended over a glass substrate. A coat of aluminum is sputtered on after the dry release in an oxygen plasma to provide good electrical conductivity for the integrated RF devices. An electrostatic deflection of $23 \mu \mathrm{m}$ has been demonstrated with an applied voltage of $5.2 \mathrm{~V}$, and has resulted in the tunable capacitor having a maximum continuous tuning ratio in excess of 4.5 to 1 . Alternative devices with a required low tuning voltage of $3 \mathrm{~V}$ have also been demonstrated to provide a tuning ratio of 2 to 1 . The power consumption of this actuator is linearly proportional to the actuation frequency, and is in the range of 10 's of nano-Watts when the device is actuated at a frequency of a few $\mathrm{kHz}$.
\end{abstract}

\section{INTRODUCTION}

Electrostatics is one of the favorable means of actuation at the reduced size scale typical of MEMS. The major attractive characteristics include simplicity in operation and device construction, low power consumption, and relatively low sensitivity to temperature variations. Various alternative actuation mechanisms have been demonstrated in MEMS, and are being used in their targeted niche applications. However, tradeoffs are evident in each of those micro actuation mechanisms. Electromagnetic actuation provides a large force because of the high energy density in magnetic materials, but is often complex to construct and integrate, and requires a significant current level (typically $\mathrm{mA}^{\prime} \mathrm{s}$ ) to operate. Electro-thermal actuation also provides a relatively large force, but in general is highly temperature dependent and requires a non-negligible amount of current to operate, as well. Depending upon the size of thermal mass and various other design parameters, electro-thermal actuators can also be limited to an operation frequency below one kilo-Hertz. Electrostatic actuators require a very low amount of current (typically $n A^{\prime}$ 's), but, in the past, often required a relatively high actuation voltage (e.g., >10 V) while still outputting a significantly smaller force. However, the low power-consumption nature of the electrostatic actuators makes it an attractive candidate for many applications, such as wireless communications, especially where the actuation force is not a critical requirement. Tunahle capacitors or varactors used in many communications circuits are an example of such applications where low power consumption is a critical requirement. By massively integrating electrostatic actuators, we have been able to lower the required electrostatic actuation voltage to approximately $5 \mathrm{~V}$, and for some designs to a voltage below $3 \mathrm{~V}$, and still maintain a required current level in the nano-Amperes range.

Tunable capacitors or varactors are widely used in $\mathrm{RF}$ communications for low-noise parametric amplifiers, harmonic frequency generators, and frequency controllers such as voltage controlled oscillators (VCO). Conventional solid-state varactors are made using either $\mathrm{p}-\mathrm{n}$ or Schottky-barrier junction structures. These solid-state components [1] often suffer from a small tuning ratio $(<30 \%)$, excessive resistive loss and thus a low Q (typically less than 10), and a low electrical self-resonance caused by large parasitics. Drastic measures are often required for the solid-state components to improve on one single parameter; for example, the use of light power to increase the tuning ratio [2]. Recent efforts within the MEMS field [3-9] have shown promising results in the realization of MEMS-based high performance tunable capacitors. Many shortcomings of solid-state varactors have indeed been overcome in these MEMS-based devices. Parameters already improved using the MEMS approaches include equivalent series resistance and $Q$ factor, capacitance tuning-ratio, associated parasitic capacitance, and intrinsic inductance and electrical selfresonance. This paper addresses the low actuation power and voltage requirement of the tunable capacitor device, and discusses in detail a massively parallel electrostatic actuator for RF MEMS applications.

\section{DEVICE DESIGN AND FABRICATION}

There are two general approaches in the MEMS-based tunable capacitor design - area-tuning interdigitated "finger" [3] and gap-tuning parallel plate [4-9] design. Each of the designs has advantages and disadvantages. A general comparison between the two was presented in [3], and the design selection is heavily guided by their targeted applications, which have very specific and often conflicting requirements on the device parameters. In general, the gap-tuning capacitors can be constructed using parallel plates stacked on top of a substrate using surface micromachining techniques. This approach provides an easy means to integrate the tunable capacitors with other conventional IC electronics. However, as in any electrostatic parallel plate system, the movable plate can only be deflected one-third of the initial gap distance before the two plates snap together as the electrostatic attracting force becomes greater than the mechanical spring back force. This deflection limitation results in a theoretical limit of $50 \%$ on the capacitance tuning range for a parallel plate tunable capacitor. Efforts have been reported in [5] to increase this $50 \%$ tuning limit to $100 \%$ by stacking three plates vertically, so that there is an actuator on each side of the middle movable plate to both increase and decrease the overall capacitance value. Nevertheless, the tuning range still comes short for many RF applications. Another inherent characteristic in a gap-tuning capacitor is the dependence of the device's RF power handling capability on the gap spacing between the two electrodes. As the capacitor is tuned higher, the gap is reduced; RF breakdown in the capacitor air-gap is thus more likely to occur. This aspect of the device must be taken into consideration when designing and using the device.

The area-tuning interdigitated "finger" capacitors do not have any theoretical tuning range limitation, as long as the fingers are designed long enough for the tuning to occur, and the springs are deflected within the material boundaries. The RF power handling capability of an area-tuning capacitor is independent of the tuned capacitance because the gap between the finger electrodes remains constant. Therefore, the area-tuning design is selected in the tunable capacitor project presented here. The interdigitated "finger" structures can be fabricated using surface micromachining technologies such as the polysilicon process. Although some thick polysilicon (e.g., $7 \mu \mathrm{m}$ ) processes have been demonstrated, typical polysilicon thickness is limited to $2 \mu \mathrm{m}$. To achieve a higher capacitance density per unit area, higher aspect 
ratio structures must be used. A silicon-on-insulator (SOI) based process was presented in [3] to allow fabrication of high aspectratio single crystal silicon structures on silicon substrate. However, substrate losses and various parasitic effects due to the use of silicon substrates have introduced a significant and negative impact to the device's RF performance. In this work, silicon substrates are consequently replaced by low-loss glass substrates, resulting in higher RF performance for the signal capacitor while maintaining similar or improved performance characteristics for the electrostatic actuator.

The device structure is a $20 \mu \mathrm{m}$ silicon layer bonded to glass substrate using epoxy as an adhesive. Figure 1 schematically illustrates the process sequence. The silicon layer is originated from a SOI substrate (fig. 1(a)) after removing the handle wafer side of an SOI along with the buried oxide. On top of the silicon device layer, $2 \mu \mathrm{m}$ aluminum is deposited (Fig. 1(b)), mainly to improve the quality factor or equivalent series resistance. Both aluminum and silicon are patterned using a single mask. The aluminum is etched by reactive ion etch (RIE) using chlorine chemistry and silicon is etched by Inductively Coupled Plasma (ICP) using the Bosch process (Fig. 1(c)). The device structure is then released in an oxygen-plasma etcher (Fig. 1(d)). After the structures are fully released, a thin layer $(\sim 0.25 \mu \mathrm{m})$ of aluminum is sputtered to further improve the quality factor (fig. I(e)). Due to the isotropic etching of epoxy, electrical isolation is naturally created between structures during the aluminum sputter deposition, and no additional mask is needed for metal patterning.

(a)

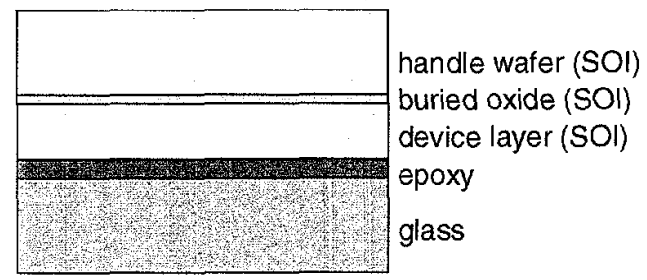

(b)

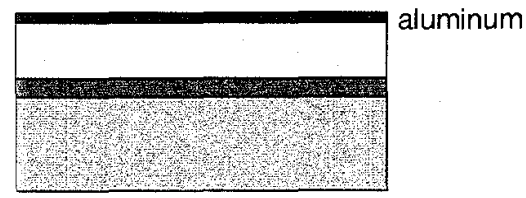

(c)

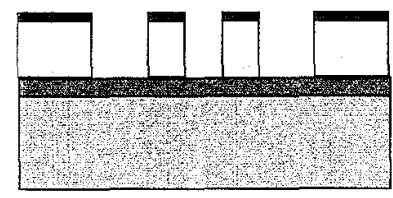

(d)

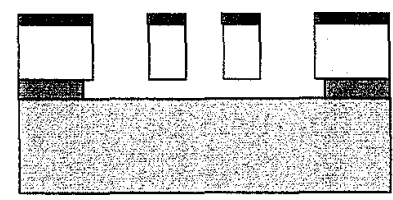

(e)

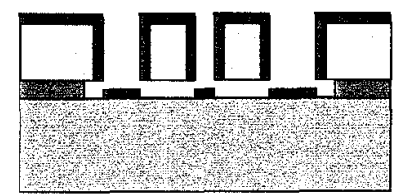

Figure 1. A schematic illustration shows the process sequence for making a single crystal silicon tunable capacitor with an integrated electrostatic actuator on a glass substrate.
Figure 2 contains three scanning electron micrographs, showing perspective views of a MEMS-based tunable capacitor on a glass substrate. The device is comprised of two mechanically joined suspended capacitors with the center suspended mechanical attachment grounded electrically. One movable capacitor is for electrostatic actuation and the other for RF signals. The mechanical attachment between the actuator and the RF capacitor enables the translation of movements provided by the actuator to the RF capacitor to realize the capacitance tuning by adjusting the physical "finger" overlapping dimension of the RF capacitor. It should be noted that the tuning can be conducted in a non-vacuum environment with a small tuning voltage of a frequency from DC up to the mechanical resonance of the structures $(\sim 10 \mathrm{kHz})$. No resonant mode of operation was used for the capacitance tuning.

(a)

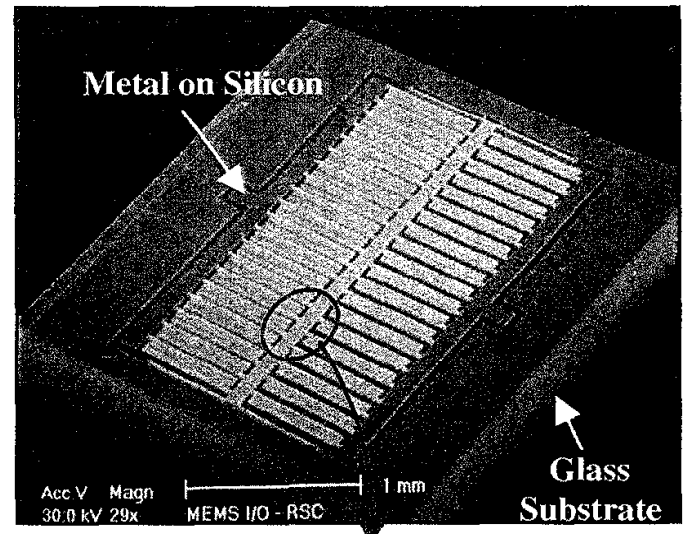

(b)
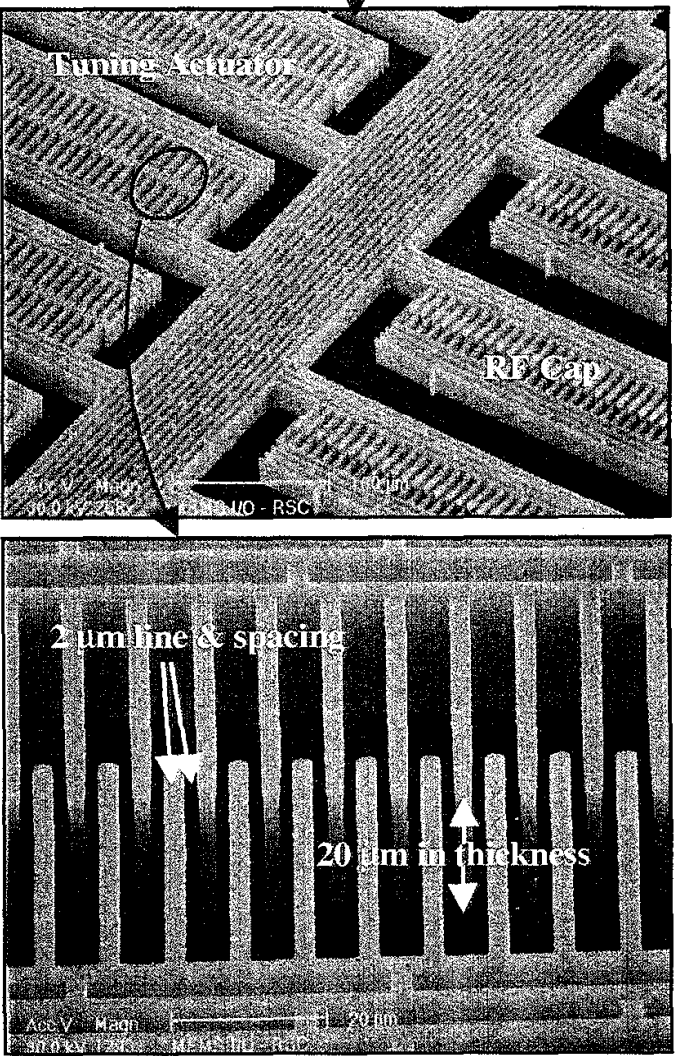

Figure 2. Scanning electron micrographs show the perspective views of a MEMS-based tunable capacitor at three different magnifications. The high aspect-ratio and the anisotropic sidewall profile provided by ICP are critical to the performance of the tunable capacitor. 
The electrostatic actuator consists of an average of 1200 sets of interdigitated fingers, which are nominally $20 \mu \mathrm{m}$ tall, $2 \mu \mathrm{m}$ in width, and $2 \mu \mathrm{m}$ spaced. These dimensions result in an actuator capacitance on the order of $2.2 \mathrm{pF}$ at $0 \mathrm{~V}$. The supporting spring designed for the device has a spring constant on the order of 0.5 $\mathrm{N} / \mathrm{m}$. The actuator is capable of providing a maximum travel distance of $30 \mu \mathrm{m}$, and occupies a nominal area of $2 \mathrm{~mm}^{2}$.

\section{EXPERIMENTAL RESULTS}

The device was first tested with a DC tuning voltage to observe visual movements. Continuous tuning has been observed by varying the tuning voltage, and no resonant operational mode is required. Figure 3 shows a top view of a portion of the tunable capacitor device with the tuning voltage at (a) $0 \mathrm{~V}$, (b) $3 \mathrm{~V}$, and (c) $5 \mathrm{~V}$. The tunable capacitor is designed to mimic a solid-state varactor diode, with its capacitance dccrcasing as the tuning voltage increases. At a zero tuning voltage, as shown in Fig. 3 (a),

(a)

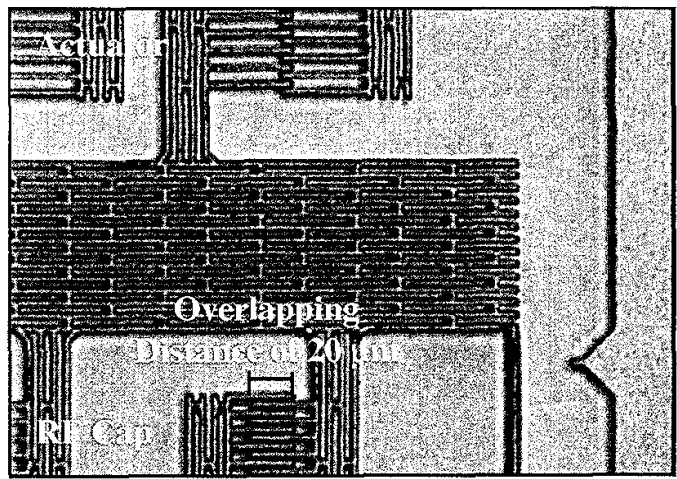

(b)

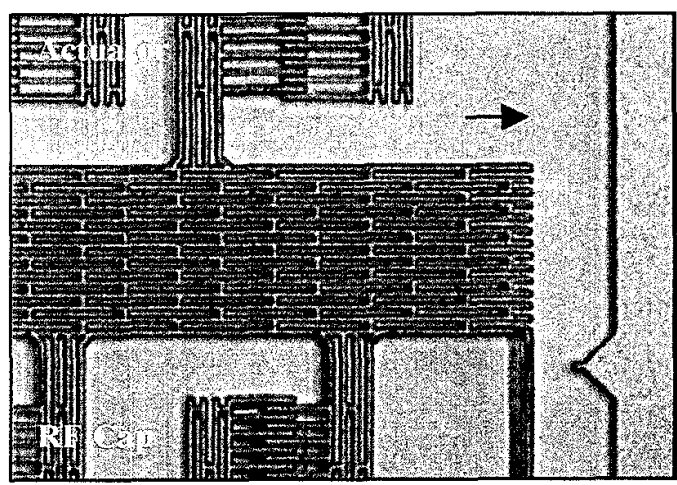

(c)

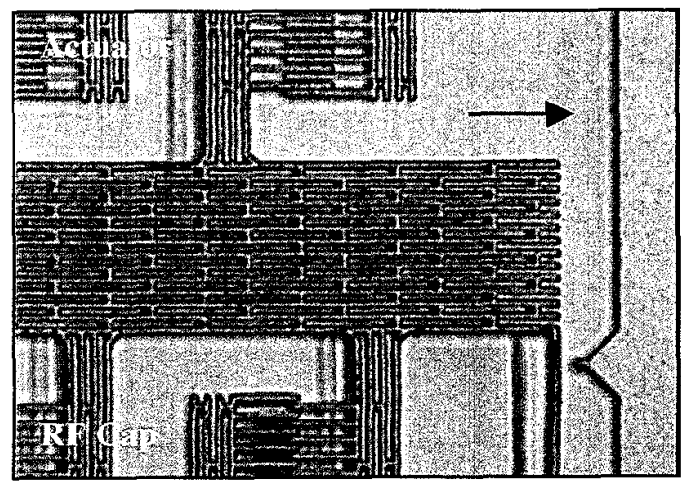

Figure 3. Top view images of a portion of the tunable capacitor (Dev. \#33) with a tuning voltage of (a) $0 \mathrm{~V}$, (b) $3 \mathrm{~V}$, and (c) $5 \mathrm{~V}$. The change in the overlapping distance between the fingers in the capacitor indicates a change in capacitance value. the overlapping distance in the RF capacitor fingers is at its maximum of $20 \mu \mathrm{m}$. This overlapping distance decreases as the tuning voltage increases, as shown in Figs. 3 (b) and (c). At a tuning voltage of $5 \mathrm{~V}$, the electrostatic actuator generates a total deflection about $20 \mu \mathrm{m}$, resulting in a zero overlapping distance in the RF capacitor tingers (Fig. 3 (c)). When further increasing the tuning voltage, the fingers in the RF capacitor become completely disengaged; the RF capacitance relies entirely on fringing fields, and decreases at a reduced rate.

The device is designed with ground-signal-ground probe pads spaced $150 \mu \mathrm{m}$ apart. RF probes with the same pitch distance were calibrated to the probe tips, using short, load, and open configurations. The capacitance was then probed using a HP8753D network analyzer from $30 \mathrm{MHz}$ to $6 \mathrm{GHz}$. All parasitic impedance associated with the tunable capacitor is included in the measurement. Figure 4 is a $\mathrm{S} 11$ plot in a Smith chart. The nominal equivalent series resistance is $1.3 \Omega$, and the electrical self-resonance is about $5 \mathrm{GHz}$ at a tuned capacitance value of $2 \mathrm{pF}$. Figure 5 contains plots of the tuned capacitance values as functions of the tuning voltage for two of the device designs. Tuning ratios of 4.55 to 1 with a maximum tuning voltage of $5.2 \mathrm{~V}$ for Dev. \#33 and 2.1 to 1 with $3 \mathrm{~V}$ for Dev. \#35 have been achicved. These

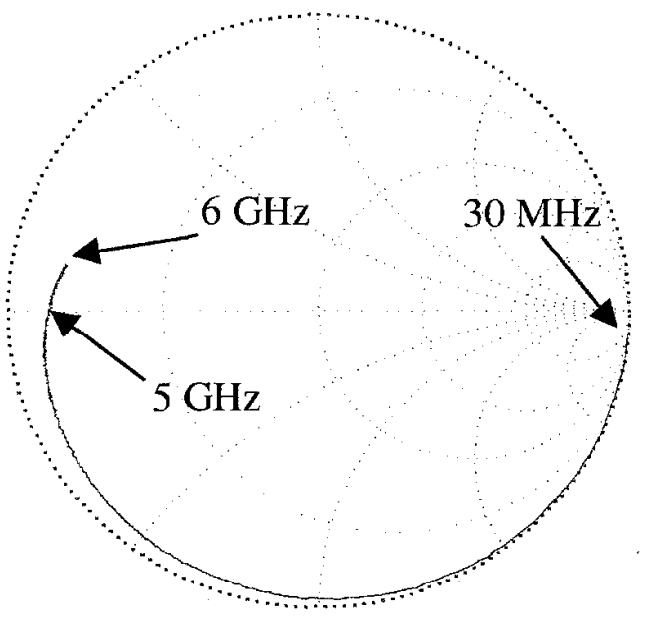

Figure 4. S11 plot in Smith chart shows a minimal parasitic effect, low equivalent series resistance, and a high self-resonance in the MEMS tunable capacitor.

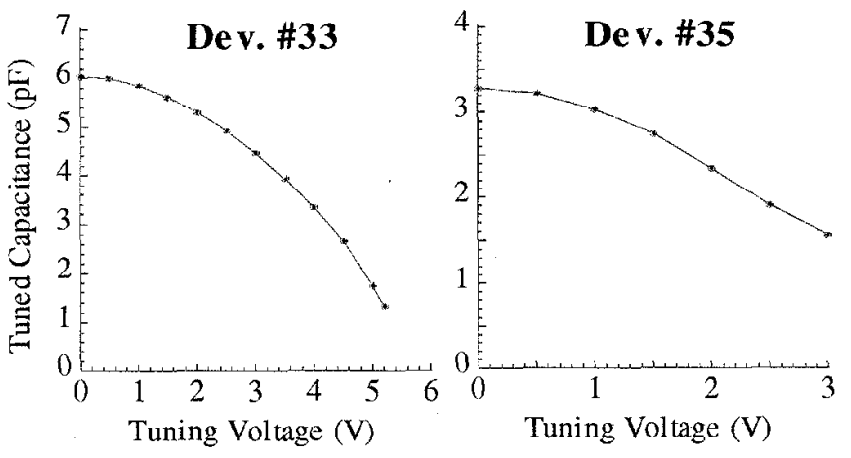

Figure 5. Tuned capacitance is plotted as functions of the applied tuning voltage. Tuning ratios of 4.55 to 1 with a maximum tuning voltage of $5.2 V$ for Dev. \#33 and 2.1 to 1 with $3 V$ for Dev. \#35 have been achieved. 
capacitance-tuning results correspond to an approximate deflection of $23 \mu \mathrm{m}$ and $8 \mu \mathrm{m}$, respectively. The approximate power required to actuate such an electrostatic actuator can be expressed as

$$
P=\frac{1}{2} C V^{2} f
$$

where $f$ is the actuation frequency. For an assumed actuation frequency of $1 \mathrm{kHz}$, the actuation power consumed by Dev. \#33 is $61 \mathrm{nW}$, with an average actuator capacitance of $4.5 \mathrm{pF}$ to achieve a full tuning range swing, which is the worst-case scenario for power consumption at a given actuation frequency.

\section{CONCLUSIONS}

A low-power and low-voltage electrostatic actuator was designed, fabricated, and demonstrated as an integrated part of a large tuning-ratio tunable capacitor for RF MEMS applications. The tunable capacitor device was made of single crystal silicon, coated with an aluminum metal thin film, on low loss glass substrate to utilize the superior mechanical properties of single crystal silicon while maintaining excellent electrical characteristics, including low parasitic effects and equivalent series resistance. The integrated electrostatic actuator has been demonstrated to provide a motion of $23 \mu \mathrm{m}$ for an applied voltage of $5.2 \mathrm{~V}(61 \mathrm{nW})$, resulting in the tunable capacitor having a continuous capacitance tuning-ratio in excess of 4.5 to 1 . Alternative devices with a required low tuning voltage of $3 \mathrm{~V}$ have also been demonstrated to provide a tuning-ratio of 2 to 1 . The power consumption of this actuator is linearly proportional to the actuation frequency, and is in the range of 10's of nano-Watts when the device is actuated at a frequency of a lew $\mathrm{kHz}$. These low power and low voltage micro-actuators have a wide range of applications, especially in the wireless arena where minimization of power consumption brings significant impacts to the overall system performance.

\section{ACKNOWLEDGEMENTS}

This work is supported by DARPA/MTO MEMS Program under BAA 96-19, Contract \# F30602-97-C-0091. The authors also express their thanks to the RSC Clean Room staff and test engineers for their help and support in the development of this project.

\section{REFERENCES}

[1] M. Soyuer, K. A. Jenkins, J. N. Burghartz, and M. D. Hulvey, "A 3V $4 \mathrm{GHz}$ nMOS Voltage-Controlled Oscillator with Integrated Resonator," Technical Digest of IEEE International Solid-State Circuit Conference, Feb. 1996, pp. 394-395

[2] D-W Kim and Y-S Kwon, "Photoresponse of Area-Variable Varactor Diode," Electronics Letters, vol. 32, no. 5, Feb. 29, 1996, pp. $466-467$

[3] J. J. Yao, S. Park, and J. DeNatale, "High Tuning-Ratio MEMS-Based Tunable Capacitors for RF Communications Applications," Proc. of 1998 Solid-State Sensor and Actuator Workshop, Hilton Head Island, 1998

[4] D. J. Young and B. E. Boser, "A Micromachined Variable Capacitor for Monolithic Low-Noise VCOs," Technical Digest of Solid State Sensor and Actuator Workshop, 1996, pp. 86-89

[5] A. Dec and K. Suyama, "Micromachined Varactor with Wide Tuning Range," Electronics Letters, vol. 33, no. 11, May 22, 1997, pp. 922-924

[6] E. Hung and S. Senturia, "Tunable Capacitors With Programmable Capacitance-Voltage Characteristic," Technical Digest of Solid State Sensor and Actuator Workshop, 1998, pp. 292-295

[7] J-H Park, H-T Kim, Y. Kwon, and Y-K Kim, "A Tunable Millimeter-Wave Filter Using Coplanar Waveguide and Micromachined Variable Capacitors," Tech Digest of The $10^{\text {th }}$ International Conference on Solid-State Sensors and Actuators, 1999, pp. 1272-1275

[8] C. L. Goldsmith, A. Malczewski, Z. J. Yao, S. Chen, J. Ehmke, and D. H. Hinzel, "RF MEMS Variable Capacitors for Tunable Filters," Int. J. of $R F$ \& Microwave Computer-Aided Engineering, vol. 9, no. 4, 1999, pp. 362-374

[9] Z. Feng, W. Zhang, B. Su, K. F. Harsh, K. C. Gupta, V. Bright, and Y. C. Lee, "Design and Modeling of RF MEMS Tunable Capacitors Using Electro-Thermal Actuators," Technical Digest of 1999 IEEE MTT-S Intemational Microwave Symposium, 1999 , pp. $1507-1510$ 